\title{
Sociologie des logiques d'action des prestataires sportifs dans un système touristique en mutation
}

\section{Clémence Perrin-Malterre}

\section{OpenEdition}

\section{Journals}

Édition électronique

URL : http://journals.openedition.org/tourisme/1135

DOI : 10.4000/tourisme.1135

ISSN : 2492-7503

Éditeur

Éditions touristiques européennes

Référence électronique

Clémence Perrin-Malterre, « Sociologie des logiques d'action des prestataires sportifs dans un système touristique en mutation », Mondes du Tourisme [En ligne], Hors-série | 2016, mis en ligne le 01 septembre 2016, consulté le 10 décembre 2020. URL : http://journals.openedition.org/tourisme/1135 ; DOI : https://doi.org/10.4000/tourisme.1135

Ce document a été généré automatiquement le 10 décembre 2020.

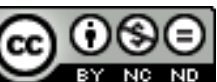

Mondes du tourisme est mis à disposition selon les termes de la licence Creative Commons Attribution - Pas d'Utilisation Commerciale - Pas de Modification 4.0 International. 


\title{
Sociologie des logiques d'action des prestataires sportifs dans un système touristique en mutation
}

\author{
Clémence Perrin-Malterre
}

\section{NOTE DE L'AUTEUR}

Ce travail a bénéficié d'une aide de l'État gérée par l'Agence nationale de la recherche au titre du programme « Investissements d'avenir » Labex ITEM - ANR-10 -LABX-50-01

1 Depuis les années 1980, des changements sont observables dans le domaine du tourisme. Il s'agit tout d'abord de l'avènement du «tourisme de masse » dans le monde (Cousin et Réau, 2011). En effet, si le tourisme est apparu dans les pays industrialisés grâce à la généralisation des congés payés permettant à la majorité de la population de voyager, celui-ci est devenu une pratique mondiale. Cependant, que ce soit à l'échelle nationale ou internationale, les inégalités en matière de départ et de consommation touristiques sont encore très fortes. Une autre tendance, essentiellement pour les pays anciennement touristiques, est celle de la personnalisation du tourisme qui repose notamment sur la composante expérientielle et qui se traduit par de nouvelles consommations touristiques axées sur l'idée d'expérience touristique coproduite par le touriste lui-même. Une autre évolution repose sur la diffusion de nouvelles valeurs dans la société comme celle du développement durable. Même si l'intégration du tourisme dans le champ du développement durable est tardive et hésitante (Clarimont et Vlès, 2008), l'avènement du tourisme durable "a permis de dépasser une vision où tourisme et développement étaient perçus comme antagonistes, pour construire une approche fondée sur la capacité de l'activité touristique à être compatible avec la durabilité " (Parra, 2010, p. 379). La diffusion de ces nouvelles valeurs s'ancre notamment dans de nouvelles modalités de consommation touristiques chez les clients. Ainsi, Marsac, Lebrun et Bouchet (2012) ont proposé un modèle d'analyse de l'importance du développement durable dans les 
expériences touristiques de la randonnée en milieu rural. Il s'avère désormais nécessaire de s'intéresser aux prestataires touristiques, et notamment d'étudier la manière dont ces évolutions affectent les pratiques professionnelles de ces acteurs de l'offre.

Dans le cadre d'un programme de recherche du Labex « Innovation dans les territoires de montagne", nous essayons de comprendre sous quelles conditions les pratiques innovantes des prestataires touristiques se traduisent par des offres pertinentes pour le marché et des retombées positives en termes de réussite individuelle mais également de valeur ajoutée pour les territoires. Nous focaliserons notre attention sur les prestataires sportifs " de nature ${ }^{1}$ » en moyenne montagne et notamment sur ceux qui proposent des offres expérientielles et ancrées dans les valeurs du développement durable.

\section{Sociologie des logiques d'action des prestataires sportifs}

3 Pour étudier les changements qui touchent les pratiques professionnelles des prestataires sportifs et touristiques, nous privilégions une approche compréhensive de trajectoires professionnelles individuelles en nous intéressant au sens que ces acteurs donnent à leurs actions. Pour cela, nous utilisons le concept de logique d'action (Amblard et alii, 1996) qui se réfère à l'articulation entre l'acteur et la situation d'action qui forme le cadre dans lequel s'inscrit l'action.

4 L'acteur se caractérise par une dimension stratégique dans le sens où il poursuit des objectifs et mobilise des ressources. Il est également doté d'une identité qui est le produit de sa trajectoire personnelle et de son enracinement social, culturel et historique. La dimension stratégique du prestataire sportif renvoie au fait qu'il est mobilisé par un ou plusieurs objectifs. Afin de les atteindre, il adoptera les comportements qui lui semblent les plus pertinents et les mieux adaptés pour réaliser son projet. Dans la mise en place de la stratégie, le prestataire sportif pourra tirer profit de l'ensemble de ses expériences antérieures et des ressources mobilisables par lui. Il développe, dans cette mesure, certains calculs qui s'inscrivent dans une rationalité limitée car le registre des choix possibles est doublement contraint: par le jeu des interactions et par le poids de la sédimentation biographique constitutive de son identité. L'acteur n'est donc pas seulement stratégique mais aussi enraciné socio-historiquement et caractérisé par sa culture et son identité, résultantes de sa trajectoire personnelle et de son appartenance à différents ensembles sociaux. À propos de la sphère du travail et de l'activité professionnelle, Amblard et alii (1996) soulignent que la dimension socio-historique de l'acteur transparaît très concrètement dans le parcours professionnel individuel et l'expérience de travail. Ces éléments sont la résultante de l'activité antérieure en tant que somme d'apprentissages qui sont autant d'éléments de structuration des manières d'être, de faire, d'analyser, d'investir les situations de travail. Il faut préciser que prendre en compte la trajectoire et le positionnement social du prestataire sportif ne signifie pas pour autant souscrire à la conception d'un déterminisme historique et structurel des biographies à partir de l'appartenance de classe. Pour Dubar (1991), la bonne compréhension de l'acteur social nécessite le positionnement du binôme choix et stratégies de l'individu sur l'articulation entre, d'une part, la culture de celui-ci (son passé et ses contraintes) et, d'autre part, ses opportunités (son présent et son libre-arbitre). Il 
convient donc de «lester l'acteur de dimensions historiques, culturelles qui sont trop rapidement exogénéisées par le raisonnement stratégique » (Amblard et alii, 1996, p. 188).

La compréhension de l'action du prestataire sportif ne peut être complète et satisfaisante si le cadre dans lequel s'inscrit l'action n'est pas pris en compte : c'est la situation d'action qui renvoie notamment au macro-environnement et à l'analyse du contexte local. L'examen du macro-environnement se focalise sur des phénomènes qui sont extérieurs au prestataire sportif, comme les contextes politique, juridique, économique, socioculturel et technologique. Cependant, limiter l'examen de ces composantes à une approche en termes de contraintes et d'opportunités objectives pour l'action n'est pas suffisant : l'étude du positionnement subjectif et des mécanismes de traduction (Callon, 1986), notamment modalisés par les dimensions symboliques et identitaires du prestataire, par rapport à ces contraintes et opportunités doit également être abordée. Il convient par conséquent d'envisager l'analyse du macro-environnement en adoptant aussi une perspective constructiviste. Il s'agit enfin de s'intéresser au contexte local dans lequel se déroule l'action. Celui-ci concerne notamment la localisation géographique de l'activité professionnelle du prestataire, mais aussi l'historique du développement touristique sur le territoire d'implantation et son poids dans l'économie locale.

En définitive, une lecture en termes de logique d'action permet d'apporter un éclairage sur les rapports complexes entre les actions stratégiques, la trajectoire et la situation de l'acteur. Par ailleurs, l'étude des logiques d'action des prestataires sportifs implique la pluralité des regards et des approches. Elle requiert l'intégration d'analyses menées à différents niveaux: non seulement à celui de l'individu en tant que tel mais également aux niveaux méso- et macro-social. Elle s'inscrit ainsi dans une démarche pluriparadigmatique qui vise le dépassement de l'opposition entre individualisme méthodologique et conception holistique de la réalité sociale.

\section{Méthodologie}

7 Dans la perspective du courant de la sociologie des logiques d'action, nous avons employé une méthodologie compréhensive. "Comprendre, c'est entendre, admettre et apprendre de cette compréhension plutôt que d'expliquer à ces mêmes acteurs pourquoi ils ne savent pas ce qu'ils font » (Amblard et alii, 1996, p. 169). Dans le cadre de nos travaux de recherche, nous avons mené des entretiens semi-directifs (Blanchet et alii, 1985) avec des prestataires sportifs implantés en moyenne montage :

- huit prestataires sportifs du Puy-de-Dôme (massif du Sancy et chaine des Puys) qui proposent des activités de découverte du milieu naturel ;

- et huit acteurs qui proposent des séjours touristiques et sportifs en moyenne montagne autour du bassin chambérien (le massif des Bauges et la Chartreuse).

8 Le choix des acteurs interrogés n'a pas été guidé par une recherche d'exhaustivité ou de représentativité de l'ensemble des activités sportives de nature proposées par les prestataires sur les territoires étudiés. Ce choix a davantage été guidé par une volonté d'interroger des professionnels qui proposent des activités douces et de découverte du milieu et qui s'inscrivent dans une démarche en lien avec le développement durable. Nous avons donc interrogés neuf accompagnateurs en moyenne montagne (AMM), quatre accompagnatrices de tourisme équestre (ATE), un moniteur de parapente, une monitrice de ski et un organisateur de séjours sportifs en pays de Savoie. 
Dans la perspective qui est la nôtre, nous sommes restés fidèles au contenu de la parole des acteurs sans chercher à discerner dans leurs propos une vérité cachée ni porter de jugement de valeur. Le guide d'entretien utilisé lors des entretiens reprend les dimensions et les indicateurs des concepts définis dans le cadre d'analyse, à savoir la dimension socio-historique et la dimension stratégique de l'acteur. Il suit une logique qui part du parcours de formation et du parcours professionnel de l'acteur pour aboutir à une description de son activité professionnelle et de ses différentes composantes. Il aborde également les composantes de la situation d'action. À partir des entretiens semi-directifs intégralement retranscrits, nous réalisons une analyse qualitative, et plus précisément une analyse thématique qui consiste à "procéder systématiquement au repérage, au regroupement et, subsidiairement, à l'examen discursif des thèmes abordés dans un corpus » (Paillé et Mucchielli, 2003, p. 162).

10 Nous avons également mené une analyse des sites internet des prestataires interrogés, car comme l'explique Guyon (2009, p. 66) à propos des accompagnateurs en montagne, «l'outil internet révèle une facette de leur stratégie de communication, mais surtout l'image professionnelle qu'ils se construisent». Ainsi, sur ces sites internet, les prestataires proposent une sélection d'objets qui expriment un point de vue singulier sur un environnement et sur les éléments qui le composent. Ce choix nous renseigne sur le rapport de l'acteur à une profession et à un espace de pratique. L'analyse de contenu des sites se base sur différentes variables: le type de photos, les logos, les verbes et les adjectifs utilisés, qui permettent de définir le positionnement du prestataire et son style de pratique professionnelle.

11 Nous allons maintenant nous intéresser aux parcours de ces prestataires sportifs menant à une professionnalisation dans l'encadrement des sports de nature.

\section{Une entrée dans le métier par le biais de parcours différenciés}

12 La majorité des acteurs interrogés ont grandi dans ou à proximité du massif où ils exercent leur activité professionnelle. Ils ont une grande pratique des sports de nature depuis leur enfance, souvent grâce à leurs parents : « Ma mère était une grande alpiniste, elle m'a emmené en montagne très très jeune " (AMM, massif des Bauges). Ainsi, ces acteurs ont d'abord été des pratiquants amateurs avant de chercher à se professionnaliser dans ce secteur (Dubois et Terral, 2013). Très vite, pour ces acteurs s'est posée la question de faire de leur passion une profession et d'encadrer des personnes dans l'apprentissage moteur. Ils ont donc rapidement passé le brevet d'État d'éducateur sportif (BEES) pour pouvoir encadrer l'activité, même si certains d'entre eux avaient débuté des études dans d'autres domaines. Ce glissement rapide vers la professionnalisation peut être interprété comme «l'expression d'une passion pour la pratique [mais aussi] comme le signe d'une vocation pour l'enseignement " (Julhe et Haschar-Noé, 2010, p. 12). Il s'agit donc d'une "posture vocationnelle» que l'on retrouve également dans l'engagement dans les métiers de la nature (Granjou et alii, 2010). Ces acteurs ont souhaité rester à la montagne : "C'était la volonté de vivre à la montagne où j'avais toujours vécu » (AMM, chaîne des Puys) ; avec souvent un fort sentiment d'attachement au territoire qui les a vus grandir : «L'attachement au lieu, il est viscéral» (ATE, massif du Sancy). Comme pour les moniteurs passionnés du Vercors, «le territoire est conçu comme un lieu de vie d'une 
qualité supérieure. Il est en même temps un espace de pratique sportive et un espace de fusion avec la nature » (Bouahouala, 2001, p. 236).

Trois autres acteurs ont opéré à un moment donné de leur carrière une reconversion dans le tourisme, après avoir été enseignant, commercial en région parisienne ou ingénieur-cadre dans une grande entreprise en région lyonnaise, avec le sentiment «d'avoir fait le tour» (AMM, Chartreuse), et aussi avec la volonté de rompre avec la vie urbaine : «Le déclic, ça a été le trop-plein de vie en région parisienne, le trop-plein de stress, de bouchons, de non-sens à ce que je faisais ; tout simplement » (AMM, massif des Bauges). Ainsi, comme pour certains moniteurs passionnés du Vercors, l'installation en moyenne montagne faisait partie d'un projet de vie, car ce territoire « représente l'antistress et l'anti-ville, il répond au besoin de liberté et à la recherche du plaisir dans le travail» (Bouhaouala, 2001, p. 236). Contrairement aux autres prestataires, ces acteurs avaient peu de vécu dans les sports de montagne. Le passage du brevet d'État a alors été vécu comme un " test ", ou comme une nécessité pour être en règle avec la législation et pouvoir emmener des personnes en milieu naturel. Mais la préparation de ce diplôme leur a aussi permis de s'immerger dans la culture de montagne.

dernier acteur se situe à l'interface de ces deux profils car il a à la fois une grande pratique dans les sports de nature, mais il n'a pas passé le brevet d'État d'éducateur sportif. En effet, après une expérience dans l'enseignement en éducation physique et sportive en région parisienne, il a créé une association qui propose des séjours touristiques en pays de Savoie: "Je voulais évoluer professionnellement dans un autre contexte que celui de l'Éducation nationale qui est parfois trop contraignant. On ne peut pas toujours faire ce que l'on veut. »

\section{Une volonté de rompre avec le tourisme de masse}

Si tous les acteurs ne l'affirment pas de manière explicite, on observe chez la majorité d'entre eux une volonté d'inscrire leur offre en dehors du tourisme de masse (Deprest, 1997) : «Je voulais sortir des valeurs du tourisme à la française dont j'ai horreur. Ce tourisme de masse, ce tourisme de parcage où on profite un maximum, où on n'est pas aimable avec le touriste, où en s'en fout plein les poches sur son dos » (AMM, Chartreuse). On retrouve ici la critique de la dérive économiste et consumériste du tourisme et des loisirs. Dans cette critique du tourisme massifié, l'opposition entre touriste et voyageur fait figure de constante (Bourdeau et Berthelot, 2009) : le touriste est considéré comme un consommateur qui profite, envahit et détruit, alors que le voyageur est celui qui découvre, échange et respecte. C'est également ce qu'exprime un accompagnateur en montagne : « Pour moi, touriste et tourisme, c'est pas un mot forcément très positif ; c'est lié à un contexte de tourisme de masse. J'aime pas ce tourisme-là. » (AMM, chaîne des Puys). Au contraire, on observe chez les prestataires interrogés une volonté de rompre avec le tourisme de masse (Zaoual, 2007) et de partager des expériences relationnelles et humaines fortes avec leurs clients, comme c'est le cas pour les éco-entrepreneurs soutenables (Corneloup, 2012) : «L'idée n'était pas de promouvoir un tourisme de masse mais plutôt de sensibiliser les visiteurs à un tourisme éducatif autour du patrimoine, des activités douces mais aussi de l'authenticité du site » (AMM, Chartreuse).

Dans ce cadre, les prestataires privilégient l'accueil de petits groupes, car, au-delà des contraintes réglementaires qui leur imposent un nombre maximal de personnes à 
encadrer, c'est aussi un moyen de favoriser les relations humaines avec leurs clients qui ne sont pas «de simples numéros comme ça peut être le cas dans les gros clubs de vacances ». (ATE, massif des Bauges). Mais aussi : « Ici, ce n'est pas l'usine comme dans les grandes stations. » (monitrice de ski, massif du Sancy)

\section{Un enrichissement expérientiel de l'offre}

Si les sports de nature en eux-mêmes sont souvent considérés comme des activités à forte valeur expérientielle (Arnould et alii, 1999; Bouchet et Lebrun, 2009 ; Ladwein, 2005), il s'agit pour les prestataires de favoriser la coproduction de l'expérience touristique :

Afin de laisser suffisamment de degrés de libertés aux consommateurs, les possibilités offertes aux opérateurs résident davantage dans la mise en place d'un dispositif de co-production de l'expérience, plutôt qu'un dispositif strict de production, qui permet d'adapter in situ la nature de l'expérience aux particularités des participants. (Ladwein, 2005, p. 117.)

18 Il s'agit alors de proposer des expériences touristiques coproduites pas le client luimême :

On a monté une prestation sur deux jours que l'on a appelée l'enquête du temps. L'idée, c'est d'aller sur le terrain avec une mallette métallique avec dedans microscope, de quoi faire un prélèvement d'échantillon, sur une tourbière, pour essayer de comprendre ce qui s'y passe. C'est une scénarisation un peu originale pour finalement un produit qui pourrait être fait d'une tout autre manière au même endroit. (AMM, massif des Bauges.)

19 Cette volonté d'impliquer et de faire participer le client de manière à ce qu'il soit pleinement acteur de ce qu'il vient vivre est également liée à une volonté de réhumanisation de la relation prestataire-client qui se traduit par de l'écoute, de l'échange, et de l'attention portée à l'autre : "Pour travailler dans le tourisme, il faut savoir écouter et partager avec les gens qui viennent chez nous; sinon, si on n'est pas capable de cela, il faut faire autre chose. " (AMM, chaîne des Puys). Cela se manifeste aussi par l'adaptation au rythme du client : «Ils sont dans un lieu qui correspond à ce qu'ils souhaitent : du calme et des activités à leur rythme. » (ATE, massif du Sancy.)

\section{Un moment d'échange et de transmission}

Le moment d'échange avec le client lors de la prestation est également un moment de transmission qui sert à faire passer des messages autour de la notion de développement durable. Mais pour la grande majorité, il s'agit d'une sensibilisation uniquement à la dimension écologique. Pour huit d'entre eux, le fait de permettre à leur clientèle de renouer le contact avec la nature semble suffire. Les autres ont la volonté de sensibiliser voire d'éduquer leurs clients : « Moi, j'aime transmettre le côté amoureux de cette nature qui est préservée ; le respect de la nature dans laquelle j'évolue au quotidien. Le respect des gens aussi." (ATE, massif du Sancy.) L'objectif est d'essayer de faire évoluer les comportements ensuite : il s'agit de «transmettre un savoir-faire au plus grand nombre pour que le grand nombre entre guillemets évolue dans sa démarche » (ATE, chaîne des Puys). Ainsi, ces prestataires touristiques utilisent «la force pédagogique des loisirs sportifs de nature dans la protection de l'environnement» (Bouhaouala et Bouchet, 2007, p. 153). Un des acteurs a créé un « centre écotouristique » qui propose des hébergements durables écologiques et un espace d'animations et de formations sur le milieu 
montagnard. On se situe ici dans la perspective des mouvements d'éducation à l'environnement (Pineau et alii, 2005) qui proposent de renouveler notre relation à la nature, celle-ci ayant «une valeur écologique et autonome (en acquérant) un droit ontologique à l'existence » (Kalaora, 2001, p. 592).

On quitte dans cette perspective le projet cartésien et humaniste de la modernité lorsqu'il s'agit de faire corps avec la nature en limitant au maximum son empreinte sur elle et en modifiant les catégories de lecture du prédateur prométhéen et contemplatif et celles du free-rider à la recherche d'une nature ludique. (Corneloup, 2011, p. 6.)

21 L'organisateur de séjours sportifs en Pays de Savoie a également travaillé « sur la mise en relation entre la théorie et la pratique du développement durable et en prenant en compte la dimension sociale qui est souvent mise de côté». En effet selon Sébastien et Brodhag (2004) la dimension sociale est la dimension «obscure » du développement durable car «si à l'origine du concept, on aspire à une certaine cohésion sociale, l'harmonie entre les êtres humains se perd peu à peu dans le magma des différentes formes d'appropriation du développement durable ». Ainsi, quelques-uns des objectifs des séjours proposés dans le domaine du vivre-ensemble sont de « développer la citoyenneté et les aptitudes sociales; développer l'esprit d'entraide, de solidarité et de coopération; de prendre du plaisir dans la vie de groupe ; ou encore de laisser libre cours à la créativité et à l'entraide (cerveau collectif) pour résoudre les problèmes ou améliorer le quotidien " (Projet séjour survie).

\section{Le développement d'un tourisme de proximité}

Les pratiques proposées sont aussi axées sur la découverte de l'environnement proche :

La balade de deux heures, même si on n'est pas trop gaillard à cheval, on se met la tête à l'envers car on a l'impression de partir super loin et en fait, on est juste à côté. Donc, tout de suite, on est en contact de la nature. Et il y a une variété de paysages : on est en montagne et après on bascule dans une forêt de hêtres. (ATE, massif du Sancy.)

23 Ainsi, les prestataires privilégient les activités de proximité afin de répondre à une certaine demande de touristes qui s'interrogent sur le bien-fondé de s'envoler au bout du monde pour retrouver le même univers qu'au coin de la rue et qui sont à la recherche d'un tourisme alternatif de proximité (Michel, 2010). Pour eux, il s'agit avant tout de découvrir l'environnement local et le patrimoine. «Je me sens comme une sorte d'agent touristique » (moniteur de parapente, chaîne des Puys). Il explique ainsi à ses clients ce qu'ils peuvent visiter dans la région. C'est ainsi que se développe un tourisme local (Amirou et Bachimon, 2000) dans lequel le client s'affirme comme un « consom'acteur du voyage », dans le respect du lieu et de ses habitants, et non plus comme un consommateur passif de circuits touristiques.

La découverte de l'environnement proche est également un moyen pour les prestataires de permettre à leurs clients de renouer un lien avec la nature (Perrin-Malterre, 2013).

Quand on part en randonnée sophrologie, on est en contact direct avec l'énergie de la nature. Et ce que j'essaye de faire, c'est d'éveiller les sens des personnes que j'accompagne, et de les rendre sensibles aux couleurs, aux odeurs, aux sons qui nous entourent. (AMM, massif des Bauges.)

C'est également ce que propose l'organisateur de séjours sportifs dans celui appelé «Épopée sauvage » : «Une expérience de "vie sauvage" pour retrouver les gestes simples 
qui nous relient à la nature» (site internet). Le contact avec la nature est « recherché comme une quête d'émotions, comme si une alliance évidente se réalisait entre l'Homme et la Nature et reconnectait à l'essentiel» (Chanvallon et Héas, 2011). Il s'agit pour les prestataires de faire aimer cette nature : «L'homme il est à sa place dans la nature; et que quand je dis l'homme, c'est un homme contemplatif qui va mieux comprendre et connaitre la nature, parce que je le répète: on protège ce que l'on aime.» On se situe alors dans la perspective d'une éthique pragmatique de l'environnement, car « à explorer les multiples raisons qui nous font attacher de la valeur à un lieu, on découvre d'autant plus d'arguments pour le protéger » (Larrère, 2010, p. 410).

\section{Le développement d'une activité économique pérenne}

L'ensemble des acteurs interrogés a pour objectif de vivre de son travail dans le domaine des sports de nature. Les prestataires natifs du lieu et qui se sont rapidement professionnalisés dans l'encadrement des sports de nature ont fait le choix d'un développement modeste et limité par peur de perdre leurs valeurs et leur personnalité du fait de l'augmentation du nombre de clients. Ces prestataires ne souhaitent pas développer leur activité davantage : «Pour ma part, c'est bien comme ça. Je ne suis pas sûre que si je développais à fond, ce serait aussi bien. » (AMM, Chartreuse.) On retrouve ici une des caractéristiques des moniteurs indépendants passionnés décrits par Bouhaouala et Chifflet (2001) dont les comportements économiques restent guidés par leur passion pour l'activité sportive. Ainsi, «ils maintiennent leur entreprise et leur volume d'activités au niveau qui leur permet l'indépendance et éviter l'assouvissement de leur passion face aux pressions économiques, velléités de rachat et tendances de croissance » (Bouhaouala, 2007). On constate chez ces prestataires une réelle volonté de ne pas rentrer dans un système mercantile où l'argent serait une fin en soi. Et cela amène parfois les acteurs à faire de nouveaux choix de carrière professionnelle :

Ma carrière professionnelle de parapente, moi, c'était pour mieux vivre, pour me sentir mieux dans ma tête ; même avoir moins de fric, puisque quand j'ai débuté, j'avais quatre fois moins de fric que quand j'avais ma paye de fonctionnaire. (Moniteur de parapente, chaîne des Puys.) Ainsi, l'argent n'est pas la finalité, c'est la qualité de vie qui prime.

Trois acteurs ont la volonté de se développer davantage, non pas pour gagner plus d'argent, mais pour développer l'emploi dans les territoires dans lesquels ils sont implantés. Ainsi, pour l'AMM qui a monté son projet d'hébergements écologiques, l'idée sous-jacente au projet était de « créer une filière locale et créer de l'emploi, de l'économie et participer à quelque chose de vertueux ». Comme c'est le cas pour les sentiers de l'imaginaire (Corneloup, 2010), on retrouve à la base de ce projet la volonté de développer une économie locale en faisant travailler des prestataires locaux pour encadrer les animations pédagogiques. Ainsi, les projets de ces prestataires ont des retombées positives sur le territoire, notamment en termes de création et de pérennisation de l'emploi : « Moi je voulais être une entreprise, parce que j'avais envie de créer du travail, de créer une richesse pour ceux qui avaient envie de me suivre." (AMM, massif des Bauges.) 


\section{Un ancrage dans le territoire}

29 professionnelle. Ils sont inscrits dans des réseaux d'acteurs touristiques et participent parfois à la vie locale. Ainsi la majorité d'entre eux sont en contact et ont créé des liens avec d'autres acteurs touristiques qui ont des activités complémentaires aux leurs (comme des hébergeurs par exemple). Mais au-delà de cela, il s'agit aussi de s'insérer dans le milieu local où se déroule l'activité professionnelle :

Dès le départ quand on a monté le projet, je suis allé voir la commune, je suis allé voir le Parc, je suis allé voir tout de suite en disant : «Voilà, aidez-moi à intégrer mon idée dans votre environnement. » (AMM, Chartreuse.)

Par ailleurs, trois prestataires sont élus dans leur commune. Un des accompagnateurs en moyenne montagne fait partie du réseau départemental «École et Nature ", réseau qui fédère les acteurs impliqués dans l'éducation à l'environnement. "C'est un réseau qui permet de rencontrer d'autres personnes, de discuter et d'échanger sur ce qu'on fait comme action d'éducation à l'environnement. » Il est donc en lien avec d'autres acteurs $\mathrm{du}$ territoire issus d'horizons divers et pas seulement du monde sportif. Ainsi, ces prestataires veulent être plus actifs dans la gestion de leur quotidien et souhaitent participer aux activités associatives et politiques de leur lieu de vie. On retrouve ici les écrits de Ray et Anderson (2001) sur les créatifs culturels qui envisagent des implications associatives et politiques dans leur vie quotidienne.

\section{Conclusion}

Les prestataires interrogés souhaitent inscrire leur offre en dehors du tourisme de masse en privilégiant notamment l'accueil de petits groupes. Il s'agit également pour eux de favoriser la coproduction de l'expérience touristique en s'inscrivant dans le cadre d'une réhumanisation de la relation commerciale. Le temps d'échange avec le client est alors envisagé comme un moment de transmission qui sert à faire passer des messages autour de la notion du développement durable. Les offres proposées sont aussi axées sur la découverte de l'environnement proche, ce qui est un moyen de permettre aux clients de renouer un lien avec la nature environnante. Enfin, pour la majorité d'entre eux, ils ont fait le choix d'un développement limité pour rester en accord avec leurs valeurs et conserver la richesse de l'échange, et font preuve d'un fort attachement au lieu qui se traduit parfois par une implication dans la vie locale.

Il s'agirait désormais de mener des observations participantes au cours de séjours proposés par les acteurs pour saisir, au-delà de leur discours, la manière concrète dont ces prestataires établissent des échanges avec leur clientèle. Ces observations participantes permettraient également d'analyser de manière fine les interactions prestataires-clients et de déterminer comment celles-ci participent au changement de regard sur la nature et au changement de comportement souhaité par les acteurs. Il semblerait également intéressant de se pencher sur les compétences nécessaires pour ces prestataires (Perrin-Malterre, 2012), la compétence pouvant «être entendue non seulement comme un ensemble de connaissances professionnelles et de savoir-faire en situation de travail mais aussi comme un ensemble de propriétés sociales des individus et un "savoir-être" au sens d'un ensemble de dispositions acquises socialement » (Gasparini 
et Pichot, 2011, p. 18). Pour les prestataires sportifs, même si la compétence technique et de sécurité reste au cœur de leur pratique professionnelle, il s'agit pour eux de développer de nouvelles compétences qui pour certaines relèvent davantage d'un ensemble de connaissances pouvant être acquises par le biais de formations initiales ou continues, tandis que d'autres relèvent davantage du savoir-faire et du savoir-être et demandent à être acquises de manière beaucoup plus autodidacte au cours de la socialisation de l'individu et tout au long de son parcours de sportif, de formation et de professionnel.

\section{BIBLIOGRAPHIE}

Henri Amblard, Philippe Bernoux, Gilles Herreros et Yves-Frédéric Livian, Les Nouvelles Approches sociologiques des organisations, Seuil, 1996.

Rachid Amirou et Philippe Bachimon (dir.), Le Tourisme local, une culture de l'exotisme, L'Harmattan, 2000.

Eric J. Arnould, Linda L. Price et Cele C. Otnes, « Making consumption magic: A study of whitewater river rafting ", Journal of Contemporary Ethnography, vol. 28, nº 1, 1999, p. 33-68.

Alain Blanchet, Hélène Bézilles-Lesquoy et Marie-France Florand, L'Entretien dans les sciences sociales : l'écoute, la parole et le sens, Dunod, 1985.

Patrick Bouchet et Anne-Marie Lebrun, Management du tourisme sportif. De la consommation à la commercialisation, « Des sociétés », Presses universitaires de Rennes, 2009.

Malek Bouhaouala, «Relations inter-entreprises dans un marché local : le cas des PE-TPE du tourisme sportif en Vercors ", Espaces et Sociétés, no 105-106, 2001, p. 229-251 [http:// gallica.bnf.fr/ark:/12148/bpt6k5619649t.image.f230.pagination].

Malek Bouhaouala, « Micromentalités et logiques d'action des entrepreneurs dirigeants de petites entreprises », Revue internationale PME, vol. 20, n² 2, 2007, p. 123-149.

Malek Bouhaouala et Patrick Bouchet, « Labellisation sectorielle et développement durable des territoires : approche théorique et opérationnelle dans le secteur du tourisme sportif de nature », Revue Gestion 2000, vol. 24, n² 2, 2007, p. 127-160.

Malek Bouhaouala et Pierre Chifflet, « Logiques d'action des moniteurs des sports de pleine nature : entre passion et profession », STAPS, $\mathrm{n}^{\circ} 56,2001$, p. 61-74.

Philippe Bourdeau et Libéra Berthelot, «La décroissance pour repenser le tourisme », L'Autre Voie , no 5, 2009 [http://www.deroutes.com/AV5/bourdeaulib5.htm].

Michel Callon, «Éléments pour une sociologie de la traduction : la domestication des coquilles Saint-Jacques et des marins-pêcheurs dans la baie de Saint-Brieuc ", L'Année Sociologique, vol. 36, 1986, p. 169-208.

Stéphanie Chanvallon et Stéphane Héas, "L'Homme et la Nature : en quête/enquête sensible », Natures Sciences Société, $\mathrm{n}^{\circ}$ 19, 2011, p. 355-364 [http://www.nss-journal.org/articles/nss/ pdf/2011/04/nss110063.pdf]. 
Sylvie Clarimont et Vincent Vlès, Tourisme durable en montagne. Entre discours et pratiques, AFNOR, 2008.

Jean Corneloup, « Les sentiers de l'imaginaire. Une créativité embryonnaire », dans Jean Corneloup et Pascal Mao (dir.), Créativité et innovation dans les loisirs sportifs de nature : un autre monde en émergence, Éditions du Fournel, 2010, p. 353-368.

Jean Corneloup, « La forme transmoderne des pratiques récréatives de nature », Développement durable et territoires, vol. 2, $\mathrm{n}^{\circ}$ 3, 2011 [http://developpementdurable.revues.org/9107].

Jean Corneloup, « Migrations géographiques et formes culturelles des pratiques récréatives », dans Niels Martin, Philippe Bourdeau et Jean-François Daller (dir.), Les Migrations d'agrément, du tourisme à l'habiter, L'Harmattan, 2012, p. 97-113.

Saskia Cousin et Bertrand Réau, « L'avènement du tourisme de masse », Les Grands Dossiers des sciences humaines, $\mathrm{n}^{\circ} 22,2011$, p. 52-55.

Florence Deprest, Enquête sur le tourisme de masse. L'écologie face au territoire, « Mappemonde », Belin, 1997.

Claude Dubar, La Socialisation. Construction des identités sociales et professionnelles, Armand Colin, 1991.

Fanny Dubois et Philippe Terral, « Les dynamiques de création d'entreprise dans le secteur du tourisme sportif », Sociologies, Théories et recherches, 2013 [http://sociologies.revues.org/4265].

William Gasparini et Lilian Pichot, « Le travail dans le sport : genèse d'un objet et perspectives interdisciplinaires ", dans William Gasparini et Lilian Pichot (dir.), Les Compétences au travail : sport et corps à l'épreuve des organisations, L'Harmattan, 2011, p. 7-35.

Céline Granjou, Isabelle Mauz et Arnaud Cosson, « Les travailleurs de la nature : une professionnalisation en tension », Sociologies, Théories et recherches, 2010 [http:// sociologies.revues.org/3296].

Frédérick Guyon, « Une accroche locale dans le réseau Internet. Les accompagnateurs en montagne ou la création de services nature-culture-sport », Téoros, vol. 28, n 2, 2009, p. 63-72 [ http://teoros.revues.org/458].

Samuel Julhe et Nadine Haschar-Noé, « Trajectoires et stratégies professionnelles dans le secteur de l'animation sportive : le cas des enseignants d'arts martiaux ", Sociétés contemporaines, $\mathrm{n}^{\circ} 77$, 2010, p. 7-29 [www.cairn.info/revue-societes-contemporaines-2010-1-page-7.htm].

Bernard Kalaora, « À la conquête de la pleine nature », Ethnologie française, vol. 31, nº 4, 2001, p. 591-597 [http://www.cairn.info/revue-ethnologie-francaise-2001-4-page-591.htm].

Richard Ladwein, « L'expérience de consommation, la mise en récit de soi et la construction identitaire : le cas du terkking ", Management et Avenir, nº 5, 2005, p. 105-118 [https:// www.cairn.info/revue-management-et-avenir-2005-3-page-105.htm].

Catherine Larrère, « Les éthiques environnementales », Natures Sciences Sociétés, vol. $18, \mathrm{n}^{\circ} 4$, 2010, p. 405-413 [https://www.cairn.info/revue-natures-sciences-societes-2010-4-page-405.htm].

Antoine Marsac, Anne-Marie Lebrun et Patrick Bouchet, « Tourisme durable et expériences touristiques : un dilemme. Proposition d'un dispositif d'analyse appliqué à l'itinérance en milieu rural », Management et Avenir, $n^{\circ}$ 56, 2012, p. 134-153 [https://www.cairn.info/revuemanagement-et-avenir-2012-6-page-134.htm].

Franck Michel, «À la recherche d'un tourisme alternatif de proximité », Espaces, nº 285, 2010, p. 14-28. 
Pierre Paillé et Alex Mucchielli, L'Analyse qualitative en sciences humaines et sociales, Armand Colin, 2012.

Constanza Parra, « Tourisme et développement durable », dans Bertrand Zuindeau (dir.), Développement durable et territoire, « Environnement et société », Presses universitaires du Septentrion, 2010, p. 375-384.

Clémence Perrin-Malterre, « Changements et évolution des pratiques professionnelles des prestataires touristiques de sports de nature ", Téoros, vol. 31, nº 1, 2012, p. 115-123 [https:// teoros.revues.org/2137].

Clémence Perrin-Malterre, « Sociologie des logiques d'action des prestataires sportifs et place de la nature dans l'offre sportive ", communication au colloque La Naturalité en mouvement, Le Pradel, IGA, université Joseph Fourier, mars 2013.

Gaston Pineau, Dominique Bachelart, Dominique Cottereau et Anne Moneyron (dir.), Habiter la Terre : écoformation terrestre pour une conscience planétaire, L'Harmattan, 2005.

Paul Ray et Sherry Ruth Anderson, Émergence des " créatifs culturels", un changement de société: enquête sur une population croissante tournée vers l'écologie, les valeurs féminines, le social et le développement psychospirituel, Yves Michel, 2001.

Léa Sébastien et Christian Brodhag, « À la recherche de la dimension sociale du développement durable », Développement durable et territoires, dossier 3, 2004, [http:// developpementdurable.revues.org/1133].

Hassan Zaoual, « Du tourisme de masse au tourisme situé : quelles transitions ? ", Marché et organisations, $\mathrm{n}^{\circ}$ 3, 2007, p. 155-182 [https://www.cairn.info/revue-marche-etorganisations-2007-1-page-155.htm].

\section{NOTES}

1. Les prestataires sportifs «de nature» sont les professionnels qui encadrent contre rémunération les « sports de nature ", vocable qui regroupe une diversité d'activités physiques et sportives terrestres, aquatiques et aériennes qui ont comme point commun de se pratiquer en milieu naturel.

\section{RÉSUMÉS}

Cet article s'intéresse aux changements qui touchent les pratiques professionnelles des prestataires touristiques des sports de nature en lien avec les mutations du système touristique. Il se situe dans une approche qui privilégie les logiques d'action des acteurs de l'offre. Pour cette étude qualitative, dix-huit prestataires sportifs exerçant leur activité en moyenne montagne ont été interrogés. Les résultats montrent que si la majorité de ces prestataires ont fait de leur passion pour les sports de nature une profession, d'autres ont opéré une reconversion professionnelle dans ce secteur d'activité. L'ensemble de ces professionnels ont fait le choix de proposer des pratiques sportives douces axées sur la découverte de l'environnement proche. 
Dans le cadre de leur prestation, ils souhaitent redonner du sens et de la valeur à l'accueil et à l'échange avec le client. Ils ont fait le choix d'un développement limité et ils font preuve d'un fort attachement au lieu.

This article focuses on changes that affect the practical of outdoor sport professional related mutations of the tourism system. This approach emphasizes logic of action of professional. In this qualitative study, eighteen outdoor sports professionals exercising their activity in mountain were interviewed. Results show that if the majority of these professionals have made a profession of their passion for outdoor sports, others have made a reconversion professional in this business. All these professionals have chosen to propose soft outdoor sports focused on the discovery of the environment. Exchange with the customer is important for them. They opted for limited economic development and they are attached with their environment.

\section{INDEX}

Mots-clés : logiques d'action, tourisme, changements, prestataires, sports de nature

Keywords : logics of action, tourism, transformation, tourism professionals, nature sports

\section{AUTEUR}

\section{CLÉMENCE PERRIN-MALTERRE}

Maître de conférences, laboratoire EDYTEM, université de Savoie - Campus scientifique, Pôle montagne - 73376 Le Bourget du Lac, Cedex - 0479758613 - clemence.malterre@univ-savoie.fr 